\title{
A Variable Metric Algorithm with Broyden Rank One Modifications for Nonlinear Equality Constraints Optimization
}

\author{
Chunyan $\mathrm{Hu}^{1}$, Zhibin $\mathrm{Zhu}^{2}$ \\ ${ }^{1}$ School of Electronic Engineering and Automation, Guilin University of Electronic Technology, Guilin, China \\ ${ }^{2}$ School of Mathematics and Computing Science, Guilin University of Electronic Technology, Guilin, China \\ Email: huchyel@hotmail.com
}

Received January 17, 2013; revised February 18, 2013; accepted March 8, 2013

\begin{abstract}
In this paper, a variable metric algorithm is proposed with Broyden rank one modifications for the equality constrained optimization. This method is viewed expansion in constrained optimization as the quasi-Newton method to unconstrained optimization. The theoretical analysis shows that local convergence can be induced under some suitable conditions. In the end, it is established an equivalent condition of superlinear convergence.
\end{abstract}

Keywords: Equality Constrained Optimization; Variable Metric Algorithm; Broyden Rank One Modification; Superlinear Convergence

\section{Introduction \& Algorithm}

This In this paper, it is proposed to consider the following nonlinear mathematical programming problem:

$$
\min f(x) \text { s.t. } g_{j}(x)=0, j \in I=\{1, \cdots, m\},
$$

where $f: R^{n} \rightarrow R, g_{j}(j \in I): R^{n} \rightarrow R$ are continuously differentiable functions. Denote the feasible set as follows:

$$
X=\left\{x \in R^{n} \mid g_{j}(x)=0, j \in I\right\} .
$$

Let $L(x, \lambda)$ be Lagrangian function of (1), and

$$
L(x, \lambda)=f(x)+\sum_{j=1}^{m} \lambda_{j} g_{j}(x) .
$$

If $\left(x_{*}, \lambda_{*}\right)$ is a KKT point pair of Equation (1), then $\nabla L x,\left.\lambda\right|_{x=x_{*}, \lambda=\lambda_{*}}=0$, i.e.,

$$
\begin{aligned}
& \nabla f\left(x_{*}\right)+\nabla g\left(x_{*}\right) \lambda_{*}=0, \\
& g\left(x_{*}\right)=0,
\end{aligned}
$$

where $g\left(x_{*}\right)=\left(g\left(x_{*}\right), j \in I\right), \nabla g\left(x_{*}\right)=\left(\nabla g\left(x_{*}\right), j \in I\right)$.

At the point pair $\left(x_{k}, \lambda_{k}\right)$, the Newton's iteration of (2) is defined as follows:

$$
\begin{aligned}
& \left(\begin{array}{cc}
\nabla_{x x}^{2} L\left(x_{k}, \lambda_{k}\right) & \nabla g\left(x_{k}\right) \\
\nabla g\left(x_{k}\right)^{\mathrm{T}} & 0
\end{array}\right)\left(\begin{array}{c}
\Delta x_{k} \\
\Delta \lambda_{k}
\end{array}\right) \\
& =-\left(\begin{array}{c}
\nabla f\left(x_{k}\right)+\nabla g\left(x_{k}\right) \lambda_{k} \\
g\left(x_{k}\right)
\end{array}\right)
\end{aligned}
$$

Later, a positive definite matrix $H_{k}^{-1}$ is replaced for $\nabla_{x x}^{2} L\left(x_{k}, \lambda_{k}\right)$ by a lot of authors to develop some kinds of variable metric methods, such as sequential quadratic programming (SQP) methods [1-5], sequential systems of linear equations (SSLE) algorithms [6-8]. In general, the computational cost of those methods is large.

In this paper, a new variable metric method is presented, in which the following fact is based on: a positive definite matrix $B_{k}$ is replaced for the matrix

$$
\left(\begin{array}{cc}
\nabla_{x x}^{2} L\left(x_{k}, \lambda_{k}\right) & \nabla g\left(x_{k}\right) \\
\nabla g\left(x_{k}\right)^{\mathrm{T}} & 0
\end{array}\right) .
$$

In the sequel, we describe the algorithm for the solution of (1). Denote

$$
\begin{gathered}
z=\left(\begin{array}{l}
x \\
\lambda
\end{array}\right), z_{k}=\left(\begin{array}{c}
x_{k} \\
\lambda_{k}
\end{array}\right), B_{k}=\left(\begin{array}{cc}
\nabla_{x x}^{2} L\left(x_{k}, \lambda_{k}\right) & \nabla g\left(x_{k}\right) \\
\nabla g\left(x_{k}\right)^{\mathrm{T}} & 0
\end{array}\right), \\
v\left(z_{k}\right)=-\left(\begin{array}{c}
\nabla f\left(x_{k}\right)+\nabla g\left(x_{k}\right) \lambda_{k} \\
g\left(x_{k}\right)
\end{array}\right), p_{k}=z_{k+1}-z_{k}=\left(\begin{array}{c}
\Delta x_{k} \\
\Delta \lambda_{k}
\end{array}\right), \\
q_{k}=v\left(z_{k+1}\right)-v\left(z_{k}\right), \\
F(z)=\|\nabla f(x)+\nabla g(x) \lambda\|^{2}+\|g(x)\|^{2} .
\end{gathered}
$$

It is obvious that $\min _{x \in X} f(x) \Leftrightarrow \min _{z \in R^{n+m}} F(z)$, and from Equation (3), we have

$$
B_{k} p_{k}=v\left(z_{k}\right) .
$$


To the system of linear Equations (4), like unconstrained optimization, $B_{k}$ is dealt with using Broyden rank one modifications as follows:

$$
B_{k+1}=B_{k}+\frac{\left(q_{k}-B_{k} p_{k}\right) p_{k}^{\mathrm{T}}}{p_{k}^{\mathrm{T}} p_{k}} .
$$

Now, the algorithm for the solution of Equation (1) can be stated as follows:

\section{Algorithm A:}

Step 1: Initialization: Given a starting point $z_{0} \in R^{n+m}$ (i.e., $x_{0} \in R^{n}, \lambda_{0} \in R^{m}$ ), and a initial positive definite matrix $B_{0} \in R^{(n+m) \times(n+m)} . \quad \varepsilon>0, k=0$;

Step 2: Compute $v\left(z_{k}\right)$. If $\left\|v\left(z_{k}\right)\right\| \leq \varepsilon$, stop;

Step 3: Compute $d_{k}=-B_{k}^{-1} v\left(z_{k}\right)$;

Step 4: Let $z_{k+1}=z_{k}+d_{k}$, and obtain $B_{k+1}$ according to (5). Set $k=k+1$. Go back to step 2 .

\section{Convergence of Algorithm}

If the algorithm stops at $z_{k}=\left(\begin{array}{c}x_{k} \\ \lambda_{k}\end{array}\right)$, then $x_{k}$ is a KKT point of (1). In the sequel, we suppose that algorithm generates an infinite sequence $\left\{z_{k}\right\}$.

Four basic assumptions are given as follows:

A1 The feasible set $X$ is nonempty; The functions $f, g_{j}(j \in I)$ are two-times continuously differentiable;

A2 For all $x \in R^{n}$, the vectors $\left\{\nabla g_{j}(x), j \in I\right\}$ are linearly independent;

A3 $\left\{x_{k}\right\}$ and $\left\{\lambda_{k}\right\}$ are bounded. There exists a KKT point pair $\left(x_{*}, \lambda_{*}\right)$, such that $\nabla_{x x}^{2} L\left(x_{*}, \lambda_{*}\right)$ is positive definite;

A4 There exists a ball $N\left(x_{*}, \delta\right)$ of radius $\delta>0$ about $\quad x_{*}$, where $\nabla^{2} f(x), \nabla^{2} g_{j}(x), \nabla g_{j}(x)(j \in I)$ satisfy the Lipschitz condition on $N\left(x_{*}, \delta\right)$.

Lemma 1 [9] Let $F: R^{n+m} \rightarrow R^{n+m}$ be continuously differentiable in some open and convex set $D$, and $F^{\prime}$ is Lipschitz continuous in $D$, then $\forall x+d \in D$, it holds that

$$
\left\|F(x+d)-F(x)-F^{\prime}(x) d\right\| \leq \frac{v}{2}\|d\|^{2},
$$

where $v$ is the Lipschitz constant. Moreover, $\forall u, v$, $x \in D$, it follows that

$$
\left\|F(u)-F(v)-F^{\prime}(u-v)\right\| \leq v \frac{\|u-x\|+\|v-x\|}{2}\|u-v\| .
$$

Lemma 2 [9] Let $F: R^{n+m} \rightarrow R^{n+m}$ be continuously differentiable in some open and convex set $D$, and $F^{\prime}$ is Lipschitz continuous in $D$. Moreover, assume $F^{\prime}(x)$ is invertible for some $x \in D$, then there exist some $\varepsilon>0, \beta>\alpha>0$, such that for all $u, v \in D$, the fact $\{\|u-x\|,\|v-x\|\} \leq \varepsilon$ implies that

$$
\alpha\|u-v\| \leq\|F(u)-F(v)\| \leq \beta\|u-v\| .
$$

Lemma 3 [9] For operator $F: R^{n+m} \rightarrow R^{n+m}$, which satisfies:

R1 $F$ is continuously differentiable on $D$;

R2 There exists a point $z_{*} \in D$, such that $F\left(z_{*}\right)=0$, and $F^{\prime}\left(z_{*}\right)$ is reversible;

R3 $F^{\prime}$ is Lipschitz continuous at $z_{*}$, i.e., there exists a constant $v$, such that

$$
\left\|F^{\prime}\left(z_{*}\right)-F^{\prime}\left(z_{*}\right)\right\| \leq v\left\|z-Z_{*}\right\|, z \in D .
$$

Let $z_{k+1}=z_{k}-H_{k}^{-1} F\left(z_{k}\right)$, where $H_{k}^{-1} \in R^{(n+m) \times(n+m)}$, and it holds that

$$
\begin{aligned}
& \left\|H_{k+1}-F^{\prime}\left(z_{*}\right)\right\| \\
& \leq\left\|H_{k}-F^{\prime}\left(z_{*}\right)\right\|+\frac{v}{2}\left(\left\|z_{k+1}-z_{*}\right\|+\left\|z_{k}-z_{*}\right\|\right), \forall k,
\end{aligned}
$$

then there exist $\varepsilon>0$ and $\delta>0$, when

$$
\left\|z_{0}-z_{*}\right\|<\varepsilon,\left\|H_{0}-F^{\prime}\left(z_{*}\right)\right\|<\delta,
$$

it is true that $z_{k+1}$ is meaning, and $z_{k}$ is linearly convergent to $Z_{*}$. Thereby, we can conclude that $Z_{k}$ is superlinearly convergent to $Z_{*}$, if and only if

$$
\lim _{k \rightarrow \infty} \frac{\left\|\left(H_{k}-F^{\prime}\left(z_{*}\right)\right)\left(z_{k+1}-z_{k}\right)\right\|}{\left\|z_{k+1}-z_{k}\right\|}=0 .
$$

In the sequel, we prove the convergence Theorem as follows:

Theorem 1 If there exist constants $\varepsilon>0$ and $\delta>0$, such that

$$
\left\|Z_{0}-Z_{*}\right\|<\varepsilon,\left\|B_{0}-v^{\prime}\left(z_{*}\right)\right\|<\delta,
$$

then $\left\{z_{k}\right\}$ is meaning, and $z_{k}$ is linearly convergent to $z_{*}$, thereby $x_{k}$ and $\lambda_{k}$ are linearly convergent to $x_{*}$ and $\lambda_{*}$ respectively.

Proof: From Lemma 3, we only prove that $v$ and $B_{k}$ satisfy conditions R1, R2, R3 and the inequality (6). From assumption A1, it is obvious that $v$ is continuously differentiable. From A3, it holds that

$$
\begin{aligned}
& v\left(z_{*}\right)=v\left(x_{*}, \lambda_{*}\right)=0, \text { and } \\
& v^{\prime}\left(z_{*}\right)=-\left(\begin{array}{cc}
\nabla^{2} f\left(x_{*}\right)+\nabla^{2} g\left(x_{*}\right) \lambda_{*} & \nabla g\left(x_{*}\right) \\
\nabla g\left(x_{*}\right)^{\mathrm{T}} & 0
\end{array}\right) .
\end{aligned}
$$

While $\nabla_{x x}^{2} L\left(x_{*}, \lambda_{*}\right)=\nabla^{2} f\left(x_{*}\right)+\nabla^{2} g\left(x_{*}\right) \lambda_{*}$ is positive definite, and $\left\{\nabla g_{j}\left(x_{*}\right), j \in I\right\}$ are linearly independent. So, it is easy to see that $v^{\prime}\left(z_{*}\right)$ is reversible. In addition,

$$
v^{\prime}\left(z_{k}\right)=-\left(\begin{array}{cc}
\nabla^{2} f\left(x_{k}\right)+\nabla^{2} g\left(x_{k}\right) \lambda_{k} & \nabla g\left(x_{k}\right) \\
\nabla g\left(x_{k}\right)^{\mathrm{T}} & 0
\end{array}\right) .
$$


From assumption A4, it holds that $v^{\prime}$ is Lipschitz continuous at $Z_{*}$. In a word, $v$ satisfies the conditions R1, R2, R3.

Now, we prove that, for $v\left(z_{*}\right)$ and $B_{k}$ generated according to (5), (6) is satisfied.

In fact, from (5), we have

$$
\begin{aligned}
& B_{k+1}-v^{\prime}\left(z_{*}\right) \\
= & B_{k}-v^{\prime}\left(z_{*}\right)+\frac{\left(q_{k}-B_{k} p_{k}\right) p_{k}^{\mathrm{T}}}{p_{k}^{\mathrm{T}} p_{k}} \\
= & B_{k}-v^{\prime}\left(z_{*}\right)+\frac{\left(v^{\prime}\left(z_{*}\right) p_{k}-B_{k} p_{k}\right) p_{k}^{\mathrm{T}}}{p_{k}^{\mathrm{T}} p_{k}} \\
& +\frac{\left(q_{k}-v^{\prime}\left(z_{*}\right) p_{k}\right) p_{k}^{\mathrm{T}}}{p_{k}^{\mathrm{T}} p_{k}} \\
= & B_{k}-v^{\prime}\left(z_{*}\right)\left(I-\frac{p_{k} p_{k}^{\mathrm{T}}}{p_{k}^{\mathrm{T}} p_{k}}\right)+\frac{\left(q_{k}-v^{\prime}\left(z_{*}\right) p_{k}\right) p_{k}^{\mathrm{T}}}{p_{k}^{\mathrm{T}} p_{k}} .
\end{aligned}
$$

So,

$$
\begin{aligned}
& \left\|B_{k+1}-v^{\prime}\left(z_{*}\right)\right\| \\
& \leq\left\|B_{k}-v^{\prime}\left(z_{*}\right)\right\|\left\|I-\frac{p_{k} p_{k}^{\mathrm{T}}}{p_{k}^{\mathrm{T}} p_{k}}\right\|+\frac{\left\|q_{k}-v^{\prime}\left(z_{*}\right) p_{k}\right\|}{\left\|p_{k}\right\|} .
\end{aligned}
$$

While

$$
\left\|I-\frac{p_{k} p_{k}^{\mathrm{T}}}{p_{k}^{\mathrm{T}} p_{k}}\right\|=\frac{p_{k}^{\mathrm{T}} p_{k}}{p_{k}^{\mathrm{T}} p_{k}}=1,
$$

and from Lemma 1, we have

$$
\begin{aligned}
& \left\|q_{k}-v^{\prime}\left(z_{*}\right) p_{k}\right\| \\
& =\left\|v\left(z_{k+1}\right)-v\left(z_{k}\right)-v^{\prime}\left(z_{*}\right)\left(z_{k+1}-z_{k}\right)\right\| \\
& \leq \frac{v}{2}\left(\left\|z_{k+1}-z_{*}\right\|+\left\|z_{k}-z_{*}\right\|\right)\left\|z_{k+1}-z_{k}\right\| .
\end{aligned}
$$

So,

$$
\left\|B_{k+1}-v^{\prime}\left(z_{*}\right)\right\| \leq\left\|B_{k}-v^{\prime}\left(z_{*}\right)\right\|+\frac{v}{2}\left(\left\|z_{k+1}-z_{*}\right\|+\left\|z_{k}-z_{*}\right\|\right),
$$

i.e., (6) is true, thereby, from Lemma 3, we get

$$
z_{k} \rightarrow z_{*} \text {,i.e., } x_{k} \rightarrow x_{*}, \lambda_{k} \rightarrow \lambda_{*} \text {. }
$$

The claim holds.

Theorem 2 Under above-mentioned assumptions, if $\varepsilon, \delta$ in Theorem 1 satisfy that

$$
6 \delta\left\|\left(v^{\prime}\left(z_{*}\right)\right)^{-1}\right\|<1,3 v \varepsilon \leq 2 \delta,
$$

then $\left\{\left(\begin{array}{l}x_{k} \\ \lambda_{k}\end{array}\right)\right\}$ is superlinearly convergent to $\left(\begin{array}{l}x_{k} \\ \lambda_{k}\end{array}\right)$, i.e.,

$$
\left\|\begin{array}{c}
x_{k+1}-x_{*} \\
\lambda_{k+1}-\lambda_{*}
\end{array}\right\|=o\left(\left\|\begin{array}{c}
x_{k}-x_{*} \\
\lambda_{k}-\lambda_{*}
\end{array}\right\|\right)
$$

Proof. From Lemma 3, we only prove that $v$ and $B_{k}$ satisfy (7). Denote $D_{k}=B_{k}-v^{\prime}\left(z_{*}\right),\|\cdot\| l_{2}$ norm, $\|\cdot\|_{F}$ Frobenius norm. From (8),

$\left\|D_{k+1}\right\|_{F} \leq\left\|D_{k}\left(I-\frac{p_{k} p_{k}^{\mathrm{T}}}{p_{k}^{\mathrm{T}} p_{k}}\right)\right\|_{F}+\frac{\left\|\left(q_{k}-v^{\prime}\left(z_{*}\right) p_{k}\right) p_{k}^{\mathrm{T}}\right\|_{F}}{p_{k}^{\mathrm{T}} p_{k}}$

Since

$$
\begin{gathered}
\left\|D_{k} \frac{p_{k} p_{k}^{\mathrm{T}}}{p_{k}^{\mathrm{T}} p_{k}}\right\|_{F}^{2}=\operatorname{tr}\left(\frac{p_{k} p_{k}^{\mathrm{T}} D_{k}^{\mathrm{T}} p_{k} p_{k}^{\mathrm{T}}}{\left(p_{k}^{\mathrm{T}} p_{k}\right)^{2}}\right) \\
=\frac{\left(D_{k} p_{k}\right)^{\mathrm{T}}\left(D_{k} p_{k}\right) p_{k}^{\mathrm{T}} p_{k}}{\left(p_{k}^{\mathrm{T}} p_{k}\right)^{2}}=\frac{\left\|D_{k} p_{k}\right\|^{2}}{\left\|p_{k}\right\|^{2}}, \\
\left\|D_{k}\right\|_{F}^{2}=\operatorname{tr}\left(D_{k}^{\mathrm{T}} D_{k}\right)=\left\|D_{k} \frac{p_{k} p_{k}^{\mathrm{T}}}{p_{k}^{\mathrm{T}} p_{k} \|_{F}^{2}}+\right\| D_{k}\left(I-\frac{p_{k} p_{k}^{\mathrm{T}}}{p_{k}^{\mathrm{T}} p_{k}}\right) \|_{F}^{2} \\
=\frac{\left\|D_{k} p_{k}\right\|^{2}}{\left\|p_{k}\right\|^{2}}+\left\|D_{k}\left(I-\frac{p_{k} p_{k}^{\mathrm{T}}}{p_{k}^{\mathrm{T}} p_{k}}\right)\right\|_{F}^{2} \cdot
\end{gathered}
$$

So,

$$
\begin{aligned}
& \left\|D_{k}\left(I-\frac{p_{k} p_{k}^{\mathrm{T}}}{p_{k}^{\mathrm{T}} p_{k}}\right)\right\|_{F}=\left(\left\|D_{k}\right\|_{F}^{2}-\frac{\left\|D_{k} p_{k}\right\|^{2}}{\left\|p_{k}\right\|^{2}}\right)^{\frac{1}{2}} \\
& \leq\left\|D_{k}\right\|_{F}-\frac{1}{2\left\|D_{k}\right\|_{F}}\left(\frac{\left\|D_{k} p_{k}\right\|}{\left\|p_{k}\right\|}\right)^{2} .
\end{aligned}
$$

In addition, from (6), (10), using the method of mathematical induction, it is not difficult to prove that $\left\|B_{k}-v^{\prime}\left(z_{*}\right)\right\| \leq\left(2-2^{-k}\right) \delta,\left\|z_{k+1}-z_{*}\right\| \leq \frac{1}{2}\left\|z_{k}-z_{*}\right\|, \forall k$,

thereby,

$$
\left\|D_{k}\right\|_{F} \leq 2 \delta, \sum_{k=0}^{\infty}\left\|z_{k}-z_{*}\right\| \leq 2 \varepsilon, \forall k .
$$

Thus, from (9), (12), (13), we have

$$
\begin{aligned}
\frac{\left\|D_{k} p_{k}\right\|^{2}}{\left\|p_{k}\right\|^{2}} \leq 2\left\|D_{k}\right\|_{F}\left(\left\|D_{k}\right\|_{F}-\left\|D_{k+1}\right\|_{F}+\frac{3}{4} v\left\|z_{k}-z_{*}\right\|\right), \\
\sum_{k=0}^{i} \frac{\left\|D_{k} p_{k}\right\|^{2}}{\left\|p_{k}\right\|^{2}} \leq 4 \delta\left(\left\|D_{0}\right\|_{F}-\left\|D_{i+1}\right\|_{F}+\frac{3}{4} v \sum_{k=0}^{i}\left\|z_{k}-z_{*}\right\|\right) \\
\leq 4 \delta^{2}+6 v \varepsilon, \forall i,
\end{aligned}
$$

i.e. $\sum_{k=0}^{i} \frac{\left\|D_{k} p_{k}\right\|^{2}}{\left\|p_{k}\right\|^{2}}$ is convergent, thereby, $\lim _{k \rightarrow \infty} \frac{\left\|D_{k} p_{k}\right\|^{2}}{\left\|p_{k}\right\|^{2}}=0$.

From Theorem 2, we don't conclude that $\left\{x_{k}\right\}$ is superlinearly convergent to $x_{*}$, i.e., $\left\|x_{k+1}-x_{*}\right\|=o\left(\left\|x_{k}-x_{*}\right\|\right)$. In the sequel, we discuss one condition which assures that $x_{k}$ is superlinearly conver- 
gent to $x_{*}$.

Lemma 4 Let $R: R^{n} \rightarrow R^{n}$ be a function defined by $R(x)=A(x)\left(\nabla f(x)+\nabla g(x) \lambda_{*}\right)+\nabla g\left(x_{*}\right) g(x)$,

where $A(x)=I_{n}-\nabla g(x)\left(\nabla g(x)^{-1} \nabla g(x)\right)^{-1} \nabla g(x)^{\mathrm{T}}$.

Then, $\left\|x_{k+1}-x_{*}\right\|=o\left(\left\|x_{k}-x_{*}\right\|\right)$, if and only if

$$
\left\|R\left(x_{k+1}\right)\right\|=o\left(\left\|x_{k+1}-x_{k}\right\|\right) \text {. }
$$

Proof. Obviously, using the triangle inequality, it holds that

$$
\left\|x_{k+1}-x_{*}\right\|=o\left(\left\|x_{k}-x_{*}\right\|\right) \Leftrightarrow\left\|x_{k+1}-x_{*}\right\|=o\left(\left\|x_{k+1}-x_{*}\right\|\right) .
$$

In addition, from $\mathrm{A} 1$, we know that $R(x)$ is continuously differentiable, and

$$
\begin{aligned}
& R\left(x_{*}\right)=0, \\
& R^{\prime}\left(x_{*}\right)=A\left(x_{*}\right) \nabla_{x x}^{2} L\left(x_{*}, \lambda_{*}\right)+\nabla g\left(x_{*}\right) \nabla g\left(x_{*}\right)^{\mathrm{T}} .
\end{aligned}
$$

It holds that $R^{\prime}\left(x_{*}\right)$ is nonsingular. In fact, let $d \neq 0$, and

$$
\left(A\left(x_{*}\right) \nabla_{x x}^{2} L\left(x_{*}, \lambda_{*}\right)+\nabla g\left(x_{*}\right) \nabla g\left(x_{*}\right)^{\mathrm{T}}\right) d=0,
$$

the facts that $\nabla g\left(x_{*}\right)$ has full rank, $A\left(x_{*}\right)$ is semi-positive definite and $\nabla_{x x}^{2} L\left(x_{*}, \lambda_{*}\right)$ is positive definite imply that

$$
A\left(x_{*}\right) \nabla_{x x}^{2} L\left(x_{*}, \lambda_{*}\right) d=0, \nabla g\left(x_{*}\right)^{\mathrm{T}} d=0 .
$$

So,

$$
A\left(x_{*}\right) d=d, d^{\mathrm{T}} \nabla_{x x}^{2} L\left(x_{*}, \lambda_{*}\right) d=0,
$$

which is a contradiction.

Thereby, from A4 and Lemma 2, there exist $\beta>\alpha>0$, such that

$$
\alpha\left\|x_{k+1}-x_{*}\right\| \leq\left\|R\left(x_{k+1}\right)\right\| \leq \beta\left\|x_{k+1}-x_{*}\right\| .
$$

So,

$$
\left\|R\left(x_{k+1}\right)\right\|=o\left(x_{k+1}-x_{k}\right) \Leftrightarrow\left\|x_{k+1}-x_{*}\right\|=o\left(\left\|x_{k+1}-x_{k}\right\|\right) .
$$

The claim holds.

Theorem 3 Under above-mentioned conditions, $\left\{x_{k}\right\}$ is superlinearly convergent to $x_{*}$ if and only if

$$
\begin{aligned}
& \| A(x)\left(\nabla f\left(x_{k}\right)+\nabla g\left(x_{k}\right) \lambda_{k}\right. \\
& \left.+\left(\nabla^{2} f\left(x_{*}\right)+\nabla^{2} g\left(x_{*}\right) \lambda_{*}\right)\left(x_{k+1}-x_{k}\right)\right) \| \\
& =o\left(\left\|x_{k+1}-x_{k}\right\|\right),
\end{aligned}
$$

and $\left\|g\left(x_{k}\right)+\nabla g\left(x_{*}\right)^{\mathrm{T}}\left(x_{k+1}-x_{k}\right)\right\|=o\left(\left\|x_{k+1}-x_{k}\right\|\right)$, i.e.,

$$
\begin{aligned}
& \left\|\left(\begin{array}{cc}
A\left(x_{k}\right) & 0_{n \times m} \\
0_{m \times n} & I_{m \times m}
\end{array}\right)\left(v\left(z_{k}\right)+v_{x}^{\prime}\left(z_{*}\right)\left(x_{k+1}-x_{k}\right)\right)\right\| \\
& =o\left(\left\|x_{k+1}-x_{k}\right\|\right) .
\end{aligned}
$$

Proof. The sufficiency: Suppose that (16) holds. We only prove that $\left\|R\left(x_{k+1}\right)\right\|=o\left(\left\|x_{k+1}-x_{k}\right\|\right)$.

From (4), we have

$$
v\left(x_{k}, \lambda_{*}\right)=B_{k} p_{k}+\left(\begin{array}{c}
\nabla g\left(x_{k}\right)\left(\lambda_{k}-\lambda_{*}\right) \\
0
\end{array}\right),
$$

So, the fact $A\left(x_{k}\right) \nabla g\left(x_{k}\right)=0$ implies that

$$
\left(\begin{array}{ll}
A\left(x_{k}\right) & 0_{n \times m}
\end{array}\right) B_{k} p_{k}-\left(\begin{array}{ll}
A\left(x_{k}\right) & 0_{n \times m}
\end{array}\right) v\left(x_{k}, \lambda_{*}\right)=0,
$$

thereby,

$$
\begin{aligned}
-R\left(x_{k+1}\right)= & \left(A\left(x_{k}\right) \quad 0_{n \times m}\right) B_{k} p_{k}-\left(R\left(x_{k+1}\right)-R\left(x_{k}\right)\right) \\
& -\nabla g\left(x_{*}\right) g\left(x_{k}\right) .
\end{aligned}
$$

From (15), we have

$$
\begin{aligned}
& R^{\prime}\left(x_{*}\right)\left(x_{k+1}-x_{k}\right) \\
& =\left(A\left(x_{*}\right) \nabla_{x x}^{2} L\left(x_{*}, \lambda_{*}\right)+\nabla g\left(x_{*}\right) \nabla g\left(x_{*}\right)^{\mathrm{T}}\right)\left(x_{k+1}-x_{k}\right) \\
& =-\left(A\left(x_{k}\right) 0_{n \times m}\right) v^{\prime}\left(z_{*}\right) p_{k}+\nabla g\left(x_{*}\right) \nabla g\left(x_{*}\right)^{\mathrm{T}}\left(x_{k+1}-x_{k}\right)
\end{aligned}
$$

So,

$$
\begin{aligned}
-R\left(x_{k+1}\right) & =\left(R\left(x_{k+1}\right)-R_{k}-R^{\prime}\left(x_{*}\right)\left(x_{k+1}-x_{k}\right)\right) \\
& -\nabla g\left(x_{*}\right)\left(g\left(x_{k}\right)+\nabla g\left(x_{*}\right)^{\mathrm{T}}\left(x_{k+1}-x_{k}\right)\right) \\
& +\left(A\left(x_{k}\right) \quad 0_{n \times m}\right) v\left(z_{k}\right) \\
& +\left(A\left(x_{k}\right) \quad 0_{n \times m}\right) v^{\prime}\left(z_{k}\right) p_{k} .
\end{aligned}
$$

While

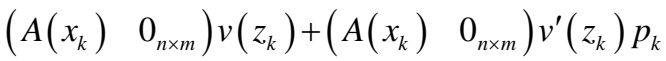

$$
\begin{aligned}
& =-A\left(x_{k}\right)\left(\nabla f\left(x_{k}\right)+\nabla g\left(x_{k}\right) \lambda_{k}\right) \\
& -A\left(x_{*}\right)\left(\nabla^{2} f\left(x_{*}\right)+\nabla^{2} g\left(x_{*}\right) \lambda_{*}\right)\left(x_{k+1}-x_{k}\right) \\
& =-A\left(x_{k}\right)\left(\nabla f\left(x_{k}\right)+\nabla g\left(x_{k}\right) \lambda_{k}\right. \\
& \left.+\left(\nabla^{2} f\left(x_{*}\right)+\nabla^{2} g\left(x_{*}\right) \lambda_{*}\right)\left(x_{k+1}-x_{k}\right)\right) \\
& +\left(A\left(x_{k}\right)-A\left(x_{*}\right)\right)\left(\nabla^{2} f\left(x_{*}\right)+\nabla^{2} g\left(x_{*}\right) \lambda_{*}\right)\left(x_{k+1}-x_{k}\right) .
\end{aligned}
$$

In addition, according to Lemma 1 , we have

$$
\left\|R\left(x_{k+1}\right)-R_{k}-R^{\prime}\left(x_{*}\right)\left(x_{k+1}-x_{k}\right)\right\|=o\left(\left\|x_{k+1}-x_{k}\right\|\right),
$$

so, from (16), it holds that $\left\|R\left(x_{k+1}\right)\right\|=o\left(\left\|x_{k+1}-x_{k}\right\|\right)$.

The necessity: Suppose that $\left\|x_{k+1}-x_{*}\right\|=o\left(\left\|x_{k}-x_{*}\right\|\right)$

i.e., $\left\|x_{k+1}-x_{*}\right\|=o\left(\left\|x_{k+1}-x_{k}\right\|\right)$.

On one hand,

$$
\begin{aligned}
& g\left(x_{k}\right)+\nabla g\left(x_{*}\right)^{\mathrm{T}}\left(x_{k+1}-x_{k}\right) \\
& =g\left(x_{k}\right)+\nabla g\left(x_{k}\right)^{\mathrm{T}}\left(x_{k+1}-x_{k}\right) \\
& \quad+\left(\nabla g\left(x_{*}\right)^{\mathrm{T}}-\nabla g\left(x_{k}\right)^{\mathrm{T}}\right)\left(x_{k+1}-x_{k}\right) .
\end{aligned}
$$


So, in order to prove that

$$
\left\|g\left(x_{k}\right)+\nabla g\left(x_{k}\right)^{\mathrm{T}}\left(x_{k+1}-x_{k}\right)\right\|=o\left(\left\|x_{k+1}-x_{k}\right\|\right) .
$$

While

$$
\begin{aligned}
\left\|g\left(x_{k}\right)+\nabla g\left(x_{k}\right)^{\mathrm{T}}\left(x_{k+1}-x_{k}\right)\right\| \\
=-\left(g\left(x_{k+1}\right)-g\left(x_{k}\right)-\nabla g\left(x_{k}\right)^{\mathrm{T}}\left(x_{k+1}-x_{k}\right)\right) \\
\quad+\left(g\left(x_{k+1}\right)-g\left(x_{*}\right)\right) .
\end{aligned}
$$

According to Lemma 1, we have

$$
\begin{aligned}
& \quad g\left(x_{k+1}\right)-g\left(x_{k}\right)-\nabla g\left(x_{k}\right)^{\mathrm{T}}\left(x_{k+1}-x_{k}\right) \\
& \quad=o\left(\left\|x_{k+1}-x_{k}\right\|\right), \\
& \left(g\left(x_{k+1}\right)-g\left(x_{k}\right)\right) \\
& \leq k_{1}\left\|x_{k+1}-x_{*}\right\|=o\left(\left\|x_{k+1}-x_{k}\right\|\right)\left(k_{1}>0\right) .
\end{aligned}
$$$$
\text { and }\left(g\left(x_{k+1}\right)-g\left(x_{k}\right)\right)
$$

So, $\left\|g\left(x_{k}\right)+\nabla g\left(x_{k}\right)^{\mathrm{T}}\left(x_{k+1}-x_{k}\right)\right\|=o\left(\left\|x_{k+1}-x_{k}\right\|\right)$.

On the other hand, the fact $A\left(x_{k}\right) \nabla g\left(x_{k}\right)=0$ implies that

$$
\begin{aligned}
& A\left(x_{k}\right)\left(\nabla f\left(x_{k}\right)+\nabla g\left(x_{k}\right) \lambda_{k}\right. \\
& \left.+\left(\nabla^{2} f\left(x_{*}\right)+\nabla^{2} g\left(x_{*}\right) \lambda_{*}\right)\left(x_{k+1}-x_{k}\right)\right) \\
& =A\left(x_{k}\right)\left(\nabla f\left(x_{k}\right)+\nabla g\left(x_{k}\right) \lambda_{*}\right. \\
& \left.+\left(\nabla^{2} f\left(x_{*}\right)+\nabla^{2} g\left(x_{*}\right) \lambda_{*}\right)\left(x_{k+1}-x_{k}\right)\right) .
\end{aligned}
$$

Let $h_{\lambda_{*}}(x)=\nabla g(x)+\nabla g(x) \lambda_{*}$, then

$$
\begin{aligned}
& \nabla f\left(x_{k}\right)+\nabla g\left(x_{k}\right) \lambda_{k}+\left(\nabla^{2} f\left(x_{*}\right)+\nabla^{2} g\left(x_{*}\right) \lambda_{*}\right)\left(x_{k+1}-x_{k}\right) \\
&=h_{\lambda_{*}}\left(x_{k}\right)+\nabla h_{\lambda_{*}}\left(x_{*}\right)^{\mathrm{T}}\left(x_{k+1}-x_{k}\right) \\
&=-\left(h_{\lambda_{*}}\left(x_{k+1}\right)-h_{\lambda_{*}}\left(x_{k}\right)-\nabla h_{\lambda_{*}}\left(x_{*}\right)^{\mathrm{T}}\left(x_{k+1}-x_{k}\right)\right) \\
&+\left(h_{\lambda_{*}}\left(x_{k+1}\right)-h_{\lambda_{*}}\left(x_{*}\right)\right) .
\end{aligned}
$$

It is easy to see that

$$
\left\|h_{\lambda_{*}}\left(x_{k}\right)+\nabla h_{\lambda_{*}}\left(x_{*}\right)^{\mathrm{T}}\left(x_{k+1}-x_{k}\right)\right\|=o\left(\left\|x_{k+1}-x_{k}\right\|\right) .
$$

Thereby,

$$
\begin{aligned}
& \| A\left(x_{k}\right)\left(\nabla f\left(x_{k}\right)+\nabla g\left(x_{k}\right) \lambda_{k}\left(\nabla f\left(x_{k}\right)+\nabla g\left(x_{k}\right) \lambda_{k}\right.\right. \\
& \left.+\left(\nabla^{2} f\left(x_{*}\right)+\nabla^{2} g\left(x_{*}\right) \lambda_{*}\right)\left(x_{k+1}-x_{k}\right)\right) \|=o\left(\left\|x_{k+1}-x_{k}\right\|\right) .
\end{aligned}
$$

The claim holds.

\section{Acknowledgements}

This work was supported in part by NNSF (No. 11061 011) of China and Guangxi Fund for Distinguished Young Scholars (2012GXSFFA060003).

\section{REFERENCES}

[1] M. J. D. Powell, “A Fast Algorithm for Nonlinearly Constrained Optimization Calculations,” In: G. A. Watson, Ed., Numerical Analysis, Springer-Verlag, Berlin, 1978, pp. 144-157. doi:10.1007/BFb0067703

[2] S.-P. Han, "Superlinearly Convergent Variable Metric Algorithm for General Nolinear Programming Problem," Mathematical Programming, Vol. 11, No. 1, 1976, pp. 263-282. doi:10.1007/BF01580395

[3] D. Q. Mayne and E. Polak, "A Superlinearly Convergent Algorithm for Constrained Optimization Problems," Mathematical Programming Studies, Vol. 16, 1982, pp. 4561. doi:10.1007/BFb0120947

[4] J.-B. Jian, C.-M. Tang, Q.-J. Hu and H.-Y. Zheng, “A Feasible Descent SQP Algorithm for General Constrained Optimization without Strict Complementarity,” Journal of Computational and Applied Mathematics, Vol. 180, No. 2, 2005, pp. 391-412. doi:10.1016/j.cam.2004.11.008

[5] Z. Jin and Y. Q. Wang, “A Simple Feasible SQP Method for Inequality Constrained Optimization with Global and Superlinear Convergence,” Journal of Computational and Applied Mathematics, Vol. 233, No. 1, 2010, pp. 30603073. doi:10.1016/j.cam.2009.11.061

[6] Y.-F. Yang, D.-H. Li and L. Q. Qi, “A Feasible Sequential Linear Equation Method for Inequality Constrained Optimization,” SIAM Journal on Optimization, Vol. 13, No. 4, 2003, pp. 1222-1244. doi:10.1137/S1052623401383881

[7] Z. B. Zhu, “An Interior Point Type QP-Free Algorithm with Superlinear Convergence for Inequality Constrained Optimization,” Applied Mathematical Modelling, Vol. 31, No. 6, 2007, pp. 1201-1212.

doi:10.1016/j.apm.2006.04.019

[8] J. B. Jian, D. L. Han and Q. J. Xu, “A New Sequential Systems of Linear Equations Algorithm of Feasible Descent for Inequality Constrained Optimization," Acta Mathematica Sinica, English Series, Vol. 26, No. 12, 2010, pp. 2399-2420. doi:10.1007/s10114-010-7432-0

[9] Y. Yuan and W. Sun, "Theory and Methods of Optimization,” Science Press, Beijing, 1997. 